\title{
BMJ
}

RESEARCH

\section{Role of co-trimoxazole prophylaxis in reducing mortality in HIV infected adults being treated for tuberculosis: randomised clinical trial}

\author{
Andrew J Nunn, associate director , ${ }^{1}$ Peter Mwaba, director and consultant physician, ${ }^{2}$ Chifumbe Chintu, \\ professor of paediatrics, ${ }^{2}$ Alwyn Mwinga, senior scientist, ${ }^{2}$ Janet $\mathrm{H}$ Darbyshire, director, ${ }^{1}$ Alimuddin Zumla, \\ professor and director, ${ }^{3}$ for the UNZA-UCLMS Project LUCOT Collaboration
}

${ }^{1}$ Medical Research Council Clinical Trials Unit, London NW1 2DA

2University Teaching Hospital, UNZA School of Medicine, Box 50110, Lusaka, Zambia

${ }^{3}$ University College London, Centre for Infectious Diseases and International Health, London W1T 4JF

Correspondence to: A Nunn ajn@ctu.mrc.ac.uk

Cite this as: $B M J$ 2008;337:a257 doi:10.1136/bmi.a257

\section{ABSTRACT}

Objective To assess the impact of prophylactic oral cotrimoxazole in reducing mortality in HIV positive Zambian adults being treated for pulmonary tuberculosis.

Design Double blind placebo controlled randomised clinical trial.

Participants Two groups of antiretroviral treatment naive adults with HIV infection: patients newly diagnosed as having tuberculosis and receiving tuberculosis treatment either for the first time or for retreatment after relapse; previously treated patients not receiving treatment. Intervention Oral co-trimoxazole or matching placebo daily.

Primary outcome measures Time to death and occurrence of serious adverse events related to study drug.

Results 1003 patients were randomised: 835 (416 cotrimoxazole, 419 placebo) were receiving treatment for tuberculosis, 762 (376 co-trimoxazole, 386 placebo) of them newly diagnosed previously untreated patients and 73 (40 co-trimoxazole, 33 placebo) receiving a retreatment regimen; 168 (84 co-trimoxazole, 84 placebo) were not on treatment but had received treatment in the past. Of 835 participants receiving tuberculosis treatment, follow-up information was available for 757 , with a total of 1012.6 person years of follow-up. A total of 310 (147 co-trimoxazole, 163 placebo) participants died, corresponding to death rates of 27.3 and 34.4 per 100 person years. In the Cox regression analysis, the hazard ratio for death (co-trimoxazole:placebo) was 0.79 (95\% confidence interval 0.63 to 0.99 ). The effect of cotrimoxazole waned with time, possibly owing to falling adherence levels; in a per protocol analysis based on patients who spent at least $90 \%$ of their time at risk supplied with study drug, the hazard ratio was 0.65 (0.45 to 0.93$)$.

Conclusions Prophylaxis with co-trimoxazole reduces mortality in HIV infected adults with pulmonary tuberculosis. Co-trimoxazole was generally safe and well tolerated.

Trial registration Current Controlled Trials ISRCTN15281875.

\section{INTRODUCTION}

Tuberculosis and HIV are leading causes of morbidity and mortality in sub-Saharan Africa. Zambia has one of the highest case notification rates of tuberculosis in the world (700 per 100000$)$, and a high proportion $(70 \%)$ of people with active tuberculosis are co-infected with HIV. ${ }^{1}$ Despite the availability of effective antituberculosis chemotherapy, mortality in HIV positive people with tuberculosis remains high. Increasing evidence indicates that this is probably due to bacterial sepsis. ${ }^{23}$ Rates of bacterial pneumonia are several times higher among HIV positive adults than in the general community.

In 1999 a randomised placebo controlled clinical trial in HIV positive patients with sputum smear positive pulmonary tuberculosis in Côte d'Ivoire found that co-trimoxazole prophylaxis significantly reduced mortality and morbidity. ${ }^{4} \mathrm{~A}$ second study in adults with symptomatic HIV disease showed significantly lower morbidity in the co-trimoxazole arm. ${ }^{5}$ These data formed the basis of a provisional recommendation by the World Health Organization and the Joint United Nations Programme on HIV/AIDS (UNAIDS) in 2000 that co-trimoxazole should be given to all adults and children in Africa with symptomatic HIV-1 infection (WHO clinical stages 2, 3, and 4). ${ }^{6}$ However, several African countries took the view that it could not be assumed that such a benefit would necessarily be applicable throughout Africa. Subsequently, a small randomised clinical trial from Senegal was stopped early (because of the announcement of the WHO/UNAIDS recommendations ${ }^{6}$ ); the authors concluded that chemoprophylaxis with low dose co-trimoxazole did not show a beneficial effect on survival or the occurrence of opportunistic or nonopportunistic infections. ${ }^{7}$ The levels of resistance of locally relevant pathogens to co-trimoxazole was low in the Côte d'Ivoire studies, whereas much higher rates have been reported elsewhere. ${ }^{8-12}$ Zambia has no policy on co-trimoxazole prophylaxis in people with HIV infection. 
After careful consideration of the available evidence, the Zambian Ministry of Health and the University of Zambia research and ethics committee agreed to three randomised placebo controlled clinical trials using cotrimoxazole prophylaxis: in children with $\mathrm{HIV}^{13}$; in postnatal women with HIV; and in adults with HIV and tuberculosis, which is reported here. Our aim was to assess the impact of prophylactic co-trimoxazole on reducing mortality in antiretroviral treatment naive adults with HIV receiving treatment for newly diagnosed active tuberculosis and in antiretroviral naive adults with HIV who had previously received antituberculosis treatment.

\section{METHODS}

Study setting and population

The setting for the study was the main referral centre for tuberculosis in Lusaka, Zambia, the University Teaching Hospital (UTH) chest clinic. We enrolled two groups of HIV positive, antiretroviral naive Zambian adults aged 16-60 years and randomised them to

Table 1| Baseline characteristics of study population (HIV positive patients newly diagnosed as having, and being treated for, tuberculosis). Values are numbers (percentages) unless stated otherwise

\begin{tabular}{|c|c|c|}
\hline Characteristics & Co-trimoxazole $(n=416)$ & Placebo $(n=419)$ \\
\hline \multicolumn{3}{|l|}{ Sex: } \\
\hline Male & $240(58)$ & $226(54)$ \\
\hline Female & $176(42)$ & $193(46)$ \\
\hline \multicolumn{3}{|l|}{ Age group (years): } \\
\hline $15-24$ & $47(11)$ & $59 *(14)$ \\
\hline $25-34$ & $210(50)$ & $211(50)$ \\
\hline $35-44$ & $127(31)$ & $122(29)$ \\
\hline$\geq 45$ & $32(8)$ & $27(6)$ \\
\hline Mean (SD) weight $(\mathrm{kg}) \dagger$ & $49.5(7.9)$ & $49.0(7.2)$ \\
\hline Mean (SD) body mass index $\ddagger$ & $15.5(4.7)$ & $15.2(4.1)$ \\
\hline \multicolumn{3}{|l|}{ HIV related symptoms: } \\
\hline Fever & $195(47)$ & $188(45)$ \\
\hline Diarrhoea & $22(5)$ & $18(4)$ \\
\hline Weight loss & $185(44)$ & $197(47)$ \\
\hline Persistent cough & $223(54)$ & $231(55)$ \\
\hline Painful genital ulceration & $12(3)$ & $13(3)$ \\
\hline Herpes zoster & $3(1)$ & $3(1)$ \\
\hline Generalised pruritic maculopapular rash & $5(1)$ & $5(1)$ \\
\hline Oral candidiasis & $16(4)$ & $22(5)$ \\
\hline Kaposi's sarcoma & $2(0.5)$ & $2(0.5)$ \\
\hline Generalised lymphadenopathy & $289(70)$ & $269(64)$ \\
\hline Other AIDS symptoms & $7(2)$ & $9(2)$ \\
\hline \multicolumn{3}{|l|}{ CD4 count (cells/10 $\left.10^{5}\right)$ : } \\
\hline $0-49$ & $20(12)$ & $25(14)$ \\
\hline $50-99$ & $28(16)$ & $22(12)$ \\
\hline 100-199 & $44(26)$ & $53(30)$ \\
\hline $200-299$ & 33 (19) & $32(18)$ \\
\hline$\geq 300$ & $46(27)$ & $45(25)$ \\
\hline
\end{tabular}

*Includes one participant aged 14 years.

†Available for 399 co-trimoxazole and 402 placebo participants.

$\ddagger$ Calculated for 396 co-trimoxazole and 397 participants.

§Based on 348 (171 co-trimoxazole, 177 placebo) participants with results no more than two weeks after randomisation. receive daily co-trimoxazole or matching placebo for the duration of the trial. The two groups were newly diagnosed, previously untreated patients with smear positive pulmonary tuberculosis receiving antituberculosis treatment (after one year, patients receiving a retreatment regimen were also eligible) and clinically healthy people previously treated for tuberculosis but no longer receiving any treatment.

All participants had a permanent address, were not pregnant, and were willing to give informed consent. We excluded patients with WHO stage 4 HIV disease who were unlikely to survive more than two weeks as well as those with a history of sulphonamide allergy and those who needed treatment with or were already receiving co-trimoxazole for other indications.

\section{Screening, informed consent, and randomisation}

All newly diagnosed, previously untreated adult patients with smear positive pulmonary tuberculosis seen routinely in a diagnostic clinic and patients who had participated in an earlier trial of tuberculosis treatment were potentially eligible. Patients were referred to the tuberculosis chest clinic at the University Teaching Hospital for assessment of their eligibility. We informed them that joining the trial would require an HIV test, the results of which would remain confidential. All participants received pre-test and post-test counselling by trained counsellors. We explained the aims and nature of the study carefully to ensure that participants understood that, in addition to the standard treatment for tuberculosis, they would be given a drug that might or might not be beneficial in preventing other sickness often seen in patients with tuberculosis and HIV. We also assessed potentially eligible participants clinically to determine any contraindications to enrolment; those with a positive HIV test result who fulfilled the other criteria for admission were taken through the consent procedure and invited to join the trial.

We used computer generated balanced randomised blocks, prepared by the MRC Clinical Trials Unit (CTU), London, to determine the randomisation schedule. An independent member of the CTU staff pre-labelled the trial drug. We entered names of eligible participants on the next available line of one of two registers (one for newly diagnosed patients, one for previously treated patients) to determine their study number and the drug supply assigned to them. None of the staff working on the trial in Zambia was aware of the allocated treatment, and at no stage was it necessary to unblind them.

We issued participants with a container of study drug bearing their trial number, gave them instructions about taking the trial tablets and what to do in the event of possible adverse reactions, and gave them an appointment date four weeks later. We provided a study identity card bearing the name of the study and the participant's study number. We encouraged participants to attend whenever they were unwell and asked them to inform the clinic if they were moving either within or out of Lusaka. 
Interventions and treatment for tuberculosis and opportunistic infections

We randomised participants in a 1:1 ratio to receive a supply of pre-labelled trial drug (co-trimoxazole or matching placebo; two tablets to be taken daily). Each co-trimoxazole tablet contained $400 \mathrm{mg}$ sulfamethoxazole and $80 \mathrm{mg}$ trimethoprim.

We followed the national tuberculosis treatment guidelines of the Ministry of Health of the government of Zambia. Patients with active, previously untreated tuberculosis received the standard regimen of ethambutol, isoniazid, rifampicin, and pyrazinamide for two months followed by isoniazid and ethambutol for six months. Patients who needed re-treatment for relapse received one of the WHO recommended retreatment regimens. $^{14}$

If participants developed any illnesses requiring the use of co-trimoxazole, we discontinued the study drug and administered open label co-trimoxazole at recommended therapeutic doses. The trial drug was resumed after treatment of an acute episode had been completed.

\section{Outcomes}

The primary end points were all cause mortality and adverse events leading to an interruption of trial drug.

\section{Follow-up}

We saw participants every four weeks up to 16 weeks and every eight weeks thereafter until the close of the trial. We gave them four-weekly supplies of 70 trial tablets up to and including the 12 week visit and two four-weekly supplies at each attendance thereafter. Patients returned unused supplies to the clinic when attending for assessment. At each visit we recorded information on the antituberculosis chemotherapy and trial drug received, together with information about visits to clinics or traditional healers or unscheduled visits to the study clinic for illness since the previous routine study attendance. We recorded details of possible adverse drug effects. When necessary, participants were admitted to hospital for investigation.

At each clinic visit, we asked participants how many doses of the trial drug they had missed and we counted pills. When participants failed to attend an outpatient

\begin{tabular}{|c|c|c|c|c|}
\hline Tuberculosis category and trial drug & $\begin{array}{l}\text { Patients } \\
\text { assessed }\end{array}$ & $\begin{array}{c}\text { Person } \\
\text { years }\end{array}$ & Deaths & $\begin{array}{l}\text { Rate }(95 \% \mathrm{Cl}) \text { per } \\
100 \text { person years }\end{array}$ \\
\hline \multicolumn{5}{|c|}{ All patients receiving tuberculosis treatment: } \\
\hline Co-trimoxazole & 416 & 538.3 & 147 & $27.3(23.2$ to 32.1$)$ \\
\hline Placebo & 419 & 474.3 & 163 & $34.4(29.5$ to 40.1$)$ \\
\hline \multicolumn{5}{|l|}{ Newly diagnosed, previously untreated: } \\
\hline Co-trimoxazole & 376 & 479.4 & 135 & $28.2(23.8$ to 33.3$)$ \\
\hline Placebo & 386 & 439.8 & 149 & $33.9(28.9$ to 39.8$)$ \\
\hline \multicolumn{5}{|l|}{ Relapse/retreatment: } \\
\hline Co-trimoxazole & 40 & 58.9 & 12 & $20.4(11.6$ to 35.8$)$ \\
\hline Placebo & 33 & 34.5 & 14 & $40.6(24.0$ to 68.5$)$ \\
\hline
\end{tabular}

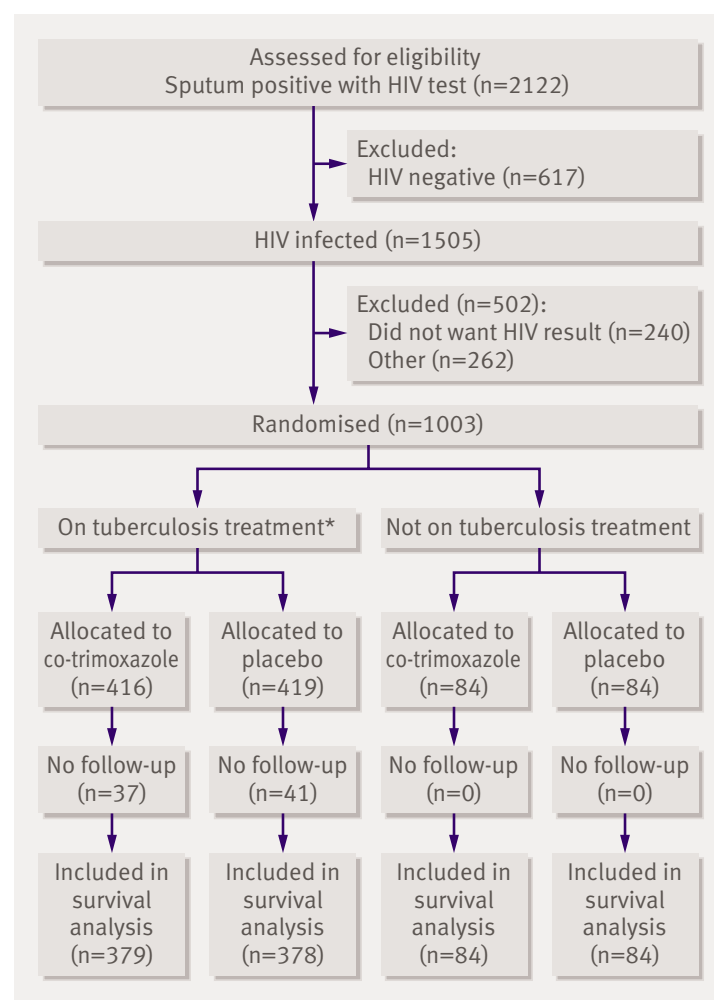

Fig 1| Trial profile. *Includes 73 (40 co-trimoxazole, 33 placebo) participants who received a retreatment regimen

appointment their names were carried forward to the next week of the diary; if they still failed to attend the home visitor team visited the home address. If they were absent from home or had moved to another address we made efforts to find them, by interviewing relatives and neighbours and visiting alternative addresses listed in the locator form. We collected clinical specimens for microbial analysis from participants who reported ill with symptoms and signs. When bacteria were cultured, we tested them for sensitivity to antibiotics.

\section{Sample size}

Assuming a death rate of 15 per 100 person years in the previously untreated participants (based on a death rate of $18 / 100$ person years in a trial in a similar population of patients in Lusaka ${ }^{15}$ ), a total of 1408 person years of observation would be needed to show a $35 \%$ reduction in death rate in patients receiving co-trimoxazole, at the $5 \%$ level of significance with $80 \%$ power; a $40 \%$ reduction would require 1045 person years.

\section{Analysis of data}

We entered all data in duplicate locally and verified them by using Epi-Info software version 6.04d. Master databases were backed up at regular intervals and copies transmitted by electronic mail to the MRC Clinical Trials Unit in London on a monthly basis.

We set up trial management committees in London and Lusaka to review the progress of the trial on a regular basis and an independent data monitoring committee to monitor the progress of the trial and 
patient safety every six to eight months. We did no formal interim analyses.

We used the Kaplan-Meier method, the log rank test, and a Cox proportional hazard regression model to make comparisons of the groups for the primary end point by time to event analyses and tested heterogeneity of survival rates in subgroups by interaction tests within Cox models. We fitted a fractional polynomial model to assess the effect of randomised group over time,${ }^{16}$ using a nearly linear pre-transformation to reduce the influence of outliers after a fixed time point. ${ }^{17}$ We made all comparisons by intention to treat and used Stata version 8 for analyses.

\section{RESULTS}

\section{Participant flow}

Figure 1 depicts the flow of participants during the study. Of 1505 people who were known to be HIV positive, 240 did not wish to receive their result; among these, 50 people did not wish to join the trial because they lived too far away, 30 reported that they needed to consult relatives, and 19 thought that their blood would be used for satanic purposes; 115 gave no reason.

\section{Study population}

Between June 2000 and January 2004, we randomised 1003 patients. Of these, 835 (416 co-trimoxazole, 419 placebo) were receiving treatment for tuberculosis, of whom 762 (376 co-trimoxazole, 386 placebo) were newly diagnosed previously untreated patients and 73 (40 co-trimoxazole, 33 placebo) were receiving a retreatment regimen; 168 (84 co-trimoxazole, 84 placebo) were not on treatment but had received treatment in the past. The trial was closed in September 2004, when funding for antiretroviral treatment became available for those participants who were eligible. Follow-up from the time of randomisation ranged from 0 to 46 months. A total of 1012.6 person years of data were accumulated from participants receiving treatment for tuberculosis at the time of randomisation (919.2 from newly diagnosed previously untreated patients and 93.4 from those being retreated for relapse) and 457.5 person years from those not on treatment. The main analyses are restricted to patients receiving antituberculosis treatment.

\section{Baseline data}

Table 1 shows the baseline characteristics of the participants receiving antituberculosis treatment at the

Table 3 |Change overtime since randomisation in possiblebenefit from co-trimoxazole overtime in HIV positive patients newly diagnosed as having, and being treated for, tuberculosis

\begin{tabular}{lcccc} 
Time period & Patients assessed & Person years & Hazard ratio $(95 \% \mathrm{Cl})$ & P value \\
All follow-up & 762 & 919.2 & $0.83(0.65$ to 1.05$)$ & 0.09 \\
\hline $0-6$ months & 762 & 315.9 & $1.24(0.81$ to 1.88$)$ & 0.3 \\
\hline 6-12 months & 546 & 225.4 & $0.55(0.34$ to 0.86$)$ & 0.009 \\
\hline $12-18$ months & 370 & 155.2 & $0.55(0.31$ to 099) & 0.05 \\
\hline$\geq 18$ months & 261 & 222.6 & $1.05(0.65$ to 1.70$)$ & 0.8 \\
\hline
\end{tabular}

time of randomisation. Most (56\%) were men, with an average age of 32.7 (SD 7.5) years; the mean weight was 49.3 (SD 7.6) kg, and the mean body mass index was 15.3 (SD 4.4). A total of 762 participants were newly diagnosed, previously untreated patients, and 73 $(8.7 \%)$ were receiving retreatment regimens. Apart from symptoms associated with tuberculosis, only a small number of participants had symptoms associated with HIV/AIDS. Only 348 (42\%) of the participants had CD4 counts (measured no later than two weeks after the start of trial drug); $55 \%$ of these had counts less than 200 cells/ $\mu$ l. The two groups were broadly similar in their characteristics.

\section{Follow-up}

We had no information on 78 (37 co-trimoxazole, 41 placebo) $(9.3 \%)$ participants after randomisation. Follow-up rates were slightly higher in the cotrimoxazole arm than in the placebo arm throughou the study. The proportion seen or known to have died remained at $80 \%$ or more throughout the study; for example, $80 \%$ of 387 participants expected at 24 months and 83\% of 266 participants expected at 30 months were assessable. During follow-up, participants in the co-trimoxazole group received trial drug during $82.9 \%$ of their time at risk and those in the placebo group received trial drug $81.0 \%$ of that time. Of those participants seen at follow-up, 269/379 (71\%) in the co-trimoxazole group and 259/378 (69\%) in the placebo group spent $80 \%$ or more of their time supplied with trial drug; the corresponding proportions who spent $90 \%$ or more of their time supplied with drug were $204 / 379(54 \%)$ and 201/378 (53\%).

\section{Outcomes and death rates in patients on tuberculosis} treatment

A total of 310 patients died during the course of the study; the death rates were 27.3 per 100 person years in participants treated with co-trimoxazole and 34.4 per 100 person years in those treated with placebo (table 2 ). Co-trimoxazole was associated with a $21 \%$ reduction in all cause mortality (hazard ratio $0.79,95 \%$ confidence interval 0.63 to $0.99 ; \mathrm{P}=0.04)$. The number needed to treat to prevent one death was 141.8 (95\% confidence interval 71.7 to 588.7 ). Figure 2 shows the KaplanMeier plots of time to death for all 835 patients. The figure suggests a delay before any benefit from cotrimoxazole becomes evident (see exploratory analyses below). We saw benefit in both newly diagnosed, previously untreated participants and in those being treated for relapse. Analysis by CD4 count on the subset of participants with data available showed no evidence of difference in benefit according to the level of immunosuppression ( $\mathrm{P}>0.5$, test for heterogeneity); we saw no difference in benefit by age or sex.

\section{Ancillary and exploratory analyses}

We explored possible changes in the effect of cotrimoxazole over a prolonged period of time by dividing time after randomisation into four segments 
- 0 -6, 6-12, 12-18, and $\geq 18$ months - in a retrospective analysis of newly diagnosed, previously untreated patients. We found significant differences in the different time periods $(\mathrm{P}=0.02$, test for heterogeneity), with no evidence of benefit in the first six months but a consistent benefit between six and 18 months associated with a $45 \%$ reduction in mortality in participants receiving co-trimoxazole (table 3). From 18 months onwards, no further benefit was apparent. Fitting a fractional polynomial to the effect of randomised group over time and shrinking the effect of outliers beyond three years confirmed these results, although the confidence limits were wide.

Unplanned analyses suggested that benefit from cotrimoxazole was associated with the proportion of time at risk for which participants were in receipt of trial drug. Thus, for those spending $90 \%$ or more, $75 \%-90 \%$, or less than $75 \%$ of their time supplied with study drug, the hazard ratios were 0.65 (0.45 to 0.93$), 0.68$ (0.40 to $1.18)$, and 1.24 (0.88 to 1.73$)$.

\section{Attendance at other clinics}

Of the 729 (362 co-trimoxazole, 367 placebo) patients who attended at least one of the follow-up clinics, 61 (17\%) co-trimoxazole participants (111 additional visits) and 92 (25\%) placebo participants (155 additional visits) reported having visited another clinic on at least one occasion between follow-up visits $(\mathrm{P}=0.006)$.

\section{Adverse events leading to planned interruption of trial drug}

Suspected adverse events leading to a planned interruption of trial drug occurred in 18 patients (12 cotrimoxazole, 6 placebo); all except six (all co-trimoxazole) resumed the trial drug. The reactions leading to permanent discontinuation were Stevens Johnson syndrome and anaemia, itchy rash, swollen face and lips, peripheral neuropathy, and two cases of anaemia.

Death rates in previously treated patients not on treatment at time of enrolment

The death rates in the 168 patients who had received treatment for tuberculosis in the past but were not on treatment at the time of enrolment were $14.5(95 \%$ confidence interval 10.3 to 20.5 ) per 100 person years in the placebo arm and 14.7 (10.5 to 20.6) per 100 in the co-trimoxazole arm.

\section{DISCUSSION}

This study confirms the results of the trial done in Côte d'Ivoire and the subsequent observational studies, in showing that prophylaxis with co-trimoxazole reduces mortality in adults with HIV infection who have pulmonary tuberculosis and that co-trimoxazole was generally safe and well tolerated. This is the only placebo controlled randomised clinical trial of cotrimoxazole prophylaxis that we are aware of in HIV positive adults with active tuberculosis since those done in Côte d'Ivoire and Senegal. ${ }^{4}$

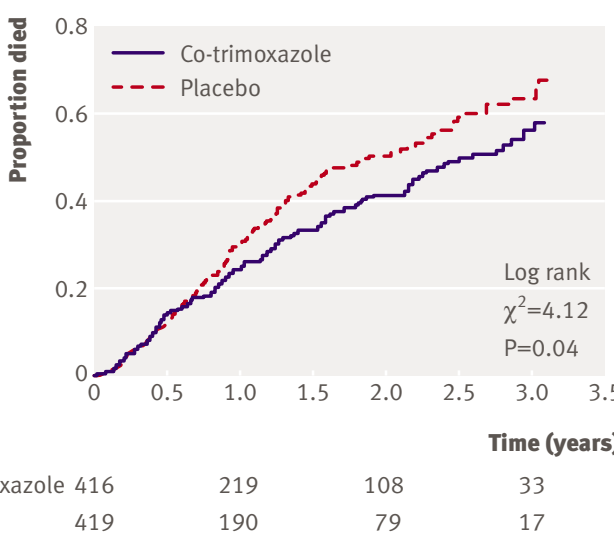

Fig 2 | Time to death according to trial drug (based on 835 HIV positive patients newly diagnosed as having, and being treated for, tuberculosis)

\section{Review of non-randomised studies}

Several non-randomised studies have been reported from central and southern Africa. ${ }^{18-21}$ A comparison of more than 3000 patients in the Hlabisa subdistrict of northern KwaZulu-Natal reported a 29\% reduction in mortality at six months when all patients with tuberculosis, irrespective of their HIV status, were given co-trimoxazole compared with a historical cohort receiving no prophylaxis. ${ }^{18}$ Zachariah and colleagues did a study in a rural district in Malawi, in which all patients with tuberculosis were offered voluntary counselling and HIV testing and those found to have HIV infection were offered co-trimoxazole adjunctive to their tuberculosis treatment. ${ }^{19}$ The relative risk of death within 12 months was decreased in those receiving co-trimoxazole (adjusted hazard ratio $0.81,95 \%$ confidence interval 0.75 to 0.87 ) compared with a historical cohort. In a study in the Karonga district of Malawi, 18 month survival rates were compared between HIV positive patients with tuberculosis registered in 1999 and those registered in 2000, who were offered co-trimoxazole. ${ }^{20}$ The hazard ratio for death for the period 3-18 months was $0.67(0.45$ to 1.00). In Uganda, co-trimoxazole was offered to a cohort of HIV positive people, and decreased mortality and morbidity were reported compared with historical controls who had not received cotrimoxazole. ${ }^{21}$ These studies have several potential limitations. As with any historical comparison, being certain that the populations were comparable in their characteristics or that levels of care did not improve over time is difficult. The extent to which mortality was ascertained may also have varied.

\section{Reduction in mortality risk}

Overall, the reduction in risk found in our study was less than has been reported elsewhere. Retrospective exploratory analyses in the newly diagnosed patients indicate a lack of benefit during the first six months, coinciding in part with the administration of rifampicin (which has a broad spectrum of activity against a variety of bacterial infections) in the initial two month 


\section{WHAT IS ALREADY KNOWN ON THIS TOPIC}

HIV positive patients with active tuberculosis have a high risk of death because of increased susceptibility to bacterial infections

Two clinical trials from Cote d'Ivoire showed that co-trimoxazole prophylaxis reduced mortality in these patients, whereas one trial from Senegal showed no effect

The findings of the west African trials might not extend to other parts of the world where levels of resistance to co-trimoxazole are high

\section{WHAT THIS STUDY ADDS}

Prophylaxis with co-trimoxazole reduced mortality in HIV positive adults with pulmonary tuberculosis in Zambia, an area with high levels of bacterial resistance

The results support UNAIDS/WHO recommendations made in 2000

intensive phase of their antituberculosis chemotherapy. After this came a period of about 12 months during which we saw a significant reduction in mortality of $45 \%$, very similar to those reported in the Cote d'Ivoire trial and the CHAP trial of HIV positive children in Zambia. ${ }^{413}$ These results should be interpreted with caution, as they are based on unplanned subgroup analyses with relatively small numbers of person years. However, the benefit seems to wane in the long term, possibly owing to a fall in adherence levels, development of resistance to co-trimoxazole, or death from causes not prevented by co-trimoxazole. An analysis according to time in receipt of study drug showed a significant benefit in patients who collected cotrimoxazole regularly.

\section{Implementation of co-trimoxazole prophylaxis}

The risks of increasing levels of bacterial resistance to co-trimoxazole and possibly increasing resistance to the antimalarial drug Fansidar (pyrimethamine-sulfadoxine) need to be assessed in the light of any benefit from giving co-trimoxazole. In vitro studies indicate cross resistance between sulfamethoxazole and sulfadoxine and between trimethoprim and pyrimethamine, ${ }^{22}$ so implementation of co-trimoxazole prophylaxis in areas where malaria and HIV overlap might result in selection for antifolate resistant malaria. Selection for resistant malaria parasites could occur within patients infected with malaria and taking co-trimoxazole prophylaxis, putting them at increased risk of treatment failure. ${ }^{2324}$ However, global policy recommendations for the use of co-artemether as prophylaxis for malaria are being increasingly implemented, and this concern may become less relevant.

The routine use of co-trimoxazole in developing countries, especially sub-Saharan Africa, has been slow to be implemented despite the provisional recommendations from WHO and UNAIDS in 2000 that cotrimoxazole should be given to everyone in Africa with HIV/AIDS, including those who have tuberculosis. The effects of current efforts to introduce highly active antiretroviral treatment into health systems in Africa will not be fully evaluated until its use is more widespread $^{25}$; it may have substantial potential to prevent fatal infections. Collaboration between national programmes on tuberculosis and $\mathrm{HIV}$ is becoming essential in optimising the joint management of tuberculosis and HIV/AIDS in highly endemic areas, making co-trimoxazole prophylaxis increasingly available.

Our findings now provide a strengthened evidence base for the UNAIDS /WHO guidelines issued in $2000{ }^{6}$ The WHO Department of HIV/AIDS has recently revised the interim UNAIDS/WHO policy on use of co-trimoxazole in people living with HIV/ AIDS. ${ }^{26}$ Analysis by CD4 counts on the subset of participants with data available showed no differences in benefit according to level of immunosuppression. Limited data on CD 4 counts suggest no clear evidence as to when to start co-trimoxazole. The optimal timing of co-trimoxazole prophylaxis has yet to be determined.

After an experts' consultation meeting in Geneva in February 2005, WHO identified four priority areas for research into co-trimoxazole prophylaxis. ${ }^{27}$ These were the role of co-trimoxazole prophylaxis in the context of concomitant antiretroviral treatment in Africa, defining the optimal timing of co-trimoxazole prophylaxis among HIV positive people with tuberculosis, defining the determinants that influence the efficacy of co-trimoxazole prophylaxis, and determining the best delivery strategies to improve the uptake of co-trimoxazole prophylaxis.

We thank the study participants, the study team members, UTH Chest Clinic staff, the Data Monitoring Committee (Tim Peto, Godfrey Biemba, Maria Quigley, and Mike Sharland), Charles Gilks for assistance with study design, the Zambian Ministry of Health national tuberculosis and leprosy control programme and the UTH Board of Management for their encouragement and support, Sarah Walker who did the exploratory analyses, and Diana Gibb of the MRC CTU for helpful comments. Contributors: AZ, AJN, JHD, and CC designed the study and obtained the funding. AM and PM carried out the study. AJN did the statistical analysis. All authors developed and edited the manuscript. AJN is the guarantor. Funding: Health and Population Division, UK Department for International Development (project grant LUCOT). The study was done under the auspices of the University of Zambia, University College London (UNZAUCLMS) Research and Training Project. The funding sponsor of the study had no role in the conception or design of the study; the collection, analysis, or interpretation of the data; or the drafting and writing of the manuscript.

Competing interests: None declared.

Ethical approval: University of Zambia research ethics committee and the joint UCL/UCLH committees on the ethics of human research, University College London.

Provenance and peer review: Not commissioned; externally peer reviewed.

1 World Health Organization. Global tuberculosis controlsurveillance, planning, financing: WHO report 2008. www.who.int/ tb/publications/global_report/2008/en/index.html.

2 Martinson NA, Karstaedt A, VenterWD, OmarT, King P, Mbengo T, et al. Causes of death in hospitalised adults with a premortem diagnosis of tuberculosis: an autopsy study. AIDS 2007:21:2043-50.

3 Nyamande K, Lalloo UG, John M. TB presenting as communityacquired pneumonia in a setting of high TB incidence and high HIV prevalence. Int J Tuberc Lung Dis 2007;12:1308-13.

4 Wiktor SZ, Sassan-Morokro M, Grant AD, Abouya L, Karon JM, Maurice C, et al. Efficacy of trimethoprim-sulphamethoxazole prophylaxis to decrease morbidity and mortality in HIV-1-infected patients with tuberculosis in Abidjan, Côte d'lvoire: a randomised controlled trial. Lancet 1999;353:1469-75.

5 Anglaret X, Chêne G, Attia A, Toure S, Lafont S, Combe P, et al. Early chemoprophylaxis with trimethoprim-sulphamethoxazole for HIV-1 infected adults in Abidjan, Côte d'Ivoire: a randomised trial. Lancet 1999;353:1463-8 
6 Joint United Nations Programme on HIV/AIDS, World Health Organization. Provisional WHO/UNAIDS Secretariat recommendations on the use of cotrimoxazole prophylaxis in adults and children living with HIV/AIDS in Africa. Geneva: UNAIDS, 2000 (available at http://data.unaids.org/Publications/IRC-pub04/ recommendation_en.pdf).

7 Maynart M, Lièvre L, Sow PS, Kony S, Gueye NF, Bassène E, et al. Primary prevention with cotrimoxazole for HIV-1-infected adults: results of the pilot study in Dakar, Senegal. J Acquir Immune Defic Syndr 2001;26:130-6.

8 Bortolotti V, Buve A. Prophylaxis of opportunistic infections in HIVinfected adults in sub-Saharan Africa: opportunities and obstacles. AIDS 2002;16:1309-17.

9 Mwansa J, Mutela K, Zulu I, Amadi B, Kelly P. Antimicrobial sensitivity in enterobacteria from AIDS patients, Zambia. Emerg Infect Dis 2002;8:92-3.

10 Zachariah R, Harries AD, Spielmann MP, Arendt V, Nchingula D, Mwenda R, et al. Changes in Escherichia coli resistance to cotrimoxazole in tuberculosis patients and in relation to co-trimoxazole prophylaxis in Thylo, Malawi. Trans R Soc Trop Med 2002;96:202-4.

11 Kassa-Kelembho E, Mbolidi CD, Service YB, Morvan J, Minssart P. Bacteraemia in adults admitted to the department of Medicine of Bangui Community Hospital (Central African Republic). Acta Trop 2003;89:67-72.

12 Gwanzura L, Pasi C, Nathoo KJ, Hakim J, Gangaidzo I, Mielke J, et al. Rapid emergence of resistance to penicillin and trimethoprimsulphamethoxazole in invasive Streptococcus pneumonia in Zimbabwe. Int J Antimicrob Agents 2003;21:557-61.

13 Chintu C, Bhat GJ, Walker AS, Mulenga V, Sinyinza F, Lishimpi K, et al. Co-trimoxazole prophylaxis against opportunistic infections in HIVinfected Zambian children (CHAP): a double blind randomised placebo-controlled trial. Lancet 2004;364:1865-71.

14 World Health Organization. Treatment of tuberculosis: guidelines for national programmes. 3rd ed. Geneva: WHO, 2003 (available at http://whqlibdoc.who.int/hq/2003/ WHO CDS TB 2003.313 eng.pdf).

15 Mwinga A, Nunn A, Ngwira B, Chintu C, Warndorff D, Fine P, et al. Mycobacterium vaccae immunotherapy as an adjunct to standard antituberculosis treatment in HIV-infected adults with pulmonary tuberculosis: a randomised placebo-controlled trial. Lancet 2002;360:1050-5.
16 Royston P, Altman DG. Regression using fractional polynomials of continuous covariates: parsimonious parametric modelling. Applied Statistics 1994;43:429-67.

17 Royston P. Multiple imputation of missing values. Stata Journal 2004;4:227-41.

18 Grimwade K, Sturm AW, Nunn Al, Mbatha D, Zungu D, Gilks CF. Effectiveness of co-trimoxazole prophylaxis on mortality in adults with tuberculosis in rural South Africa. AIDS 2005;19:163-8.

19 Zachariah R, Spielmann MP, Chinji C, Gomani P, Arendt V, Hargreaves NJ, et al. Voluntary counselling, HIV testing and adjunctive co-trimoxazole reduces mortality in tuberculosis patients in Thyolo, Malawi. AIDS 2003;17:1053-61.

20 Mwaungulu FB, Floyd S, Crampin AC, Kasimba S, Malema S, Kanyongoloka $\mathrm{H}$, et al. Co-trimoxazole prophylaxis reduces mortality in human immunodeficiency virus-positive tuberculosis patients in Karonga District, Malawi. Bull World Health Organ 2004:82:354-63.

21 Mermin J, Lule J, Ekwaru JP, Malamba S, Downing R, Ransom R, et al. Effect of co-trimoxazole prophylaxis on morbidity, mortality, CD4 cell count and viral load in HIV infection in rural Uganda. Lancet 2004:364:1428-34

22 Iyer JK, Milhous WK, Cortese JF, Kublin JG, Plowe CV. Plasmodium falciparum cross-resistance between trimethoprim and pyrimethamine. Lancet 2001;358:1066-7.

23 Malamba SS, Mermin J, Reingold A, Lule JR, Downing R, Ransom R, et al. Effect of co-trimoxazole prophylaxis taken by HIV-infected persons on the selection of sulfadoxine-pyrimethamine-resistant malaria-resistant parasites among HIV-uninfected household members. Am J Trop Med Hyg 2006;75:375-80.

24 Laufer MK, Plowe CV. Co-trimoxazole prophylaxis and malaria in Africa: have the important questions been answered? Am / Trop Med Hyg 2006;75:373-4.

25 Murray J. What to do until HAART arrives? Int J Tuberc Lung Dis 2000;6:1035-7.

26 World Health Organization. WHO expert consultation on cotrimoxazole prophylaxis in HIV infection. Geneva: WHO 2006. (available at www.who.int/hiv/pub/meetingreports/ctx/en/).

27 World Health Organization. TB/HIV research priorities in resourcelimited settings: report of an expert consultation. Geneva: WHO, 2005 (available at http://whqlibdoc.who.int/hq/2005/ WHO HTM TB 2005.355.pdf).

Accepted: 9 May 2008 